\title{
Measuring Physician Quality and Efficiency in an Era of Practice Transformation: PCMH as a Case Study
}

Lewis G. Sandy, $M D^{1}$

Heidi Haltson, BS ${ }^{2}$

Brent A. Metfessel, $M D^{1}$

Conway Reese, $\mathrm{BS}^{2}$

'UnitedHealth Group, Minnetonka, Minnesota

${ }^{2}$ Optum, Eden Prairie, Minnesota
Conflicts of interest: authors are employees of UnitedHealth Group.

\section{CORRESPONDING AUTHOR}

Lewis G. Sandy, MD

UnitedHealth Group

9900 Bren Rd E

Minnetonka, MN 55343

lewis_g_sandy@uhg.com

\begin{abstract}
Practicing physicians face myriad challenges as health care undergoes considerable transformation, including advancing efforts to measure and report on physician quality and efficiency, as well as the growth of new care models such as Accountable Care Organizations and patient-centered medical homes (PCMHs). How do these transformational forces relate to one another? How should practicing physicians focus and prioritize their improvement efforts? This Special Report examines how physicians' performance on quality and efficiency measures may interact with delivery reforms, focusing on the $\mathrm{PCMH}$. We note that although the $\mathrm{PCMH}$ is a promising model, published evidence is mixed. Using data and experience from a large commercial insurer's performance transparency and PCMH programs, we further report that longitudinal analysis of UnitedHealthcare's PCMH program experience has shown favorable changes; however, cross-sectional analysis indicates that National Committee for Quality Assurance's PCMH designation is positively associated with achieving program Quality benchmarks, but negatively associated with program Efficiency benchmarks. This example illustrates some key issues for physicians in the current environment, and we provide suggestions for physicians and other stakeholders on understanding and acting on information from physician performance measurement programs.
\end{abstract}

Ann Fam Med 2015;13:264-268. doi: 10.1370/afm.1784.

\section{INTRODUCTION}

$\mathrm{P}$ racticing physicians face myriad challenges as health care undergoes considerable transformations. Among these transformations, efforts to analyze and report on physician-level measures of quality and efficiency are growing rapidly. Simultaneously, there is growth of new payment and delivery models such as Accountable Care Organizations and patient-centered medical homes (PCMHs). How do these forces of change relate to one another? Does the measurement and reporting of quality and efficiency facilitate practice transformation or impede it? Do practice transformations lead to better or worse scores on quality and efficiency of care, and if so, how? And what are the implications of these 2 related trends on physicians and their patients?

\section{OVERVIEW OF PHYSICIAN QUALITY AND EFFICIENCY MEASUREMENT AND REPORTING}

Following the mantra "you can't improve what you don't measure," both the public and the private sectors have accelerated programs to measure and publicly report on the quality and efficiency of physician services. Although a full description of such efforts is beyond the scope of this article, some of the more prominent initiatives include programs from the Centers for Medicare and Medicaid Services such as the Physician Quality Reporting System and the forthcoming Value-Based Payment Modifier program; local and regional measurement and reporting collaboratives such as Minnesota Community Measurement, the Aligning Forces for Quality supported by the Robert Wood Johnson Foundation, and the Net- 
work for Regional Health Improvement; and programs from private payers such as the Blue Cross and Blue Shield of Massachusetts Alternative Quality Contract and the UnitedHealth Premium Designation Program.

These measurement and reporting programs, while varying in their focus and approach, generally rely on claims-based measures of quality and efficiency because of the wide availability of claims data for analysis, the ability to achieve larger sample sizes, advances in analytic methods such as episode groupers, and lower administrative costs compared with measures requiring chart abstraction. And although the federal Meaningful Use program has provided incentives for physicians to adopt electronic health record (EHR) systems that can report on quality measures, issues of reliability, validity, and feasibility of EHR-based reporting continue to be substantial. ${ }^{2}$

The stakes thus are high and growing greater for practicing physicians as they consider how they are being measured, as well as how to approach the emerging new models for care delivery and payment. Primary care physicians in particular need to focus on how the PCMH might relate to physician-level quality and efficiency measurement. The PCMH model includes primary care transformation using teams and proactive care plans; enhanced access and care coordination; and a systems-based approach to whole-person care. It also includes a new payment model—typically a blended payment program that includes fee-forservice payments, a care management fee that supports the enhanced services in the model, and performancebased bonuses or other enhanced reimbursement. ${ }^{3}$

Many demonstration projects rely heavily on criteria developed by the National Committee for Quality Assurance (NCQA), which has developed a PCMH Recognition Program. As of May 1, 2013, more than 26,634 clinicians had achieved this recognition, which is based on specific standards in 6 areas: enhanced access and continuity; identification and management of patient populations; planning and management of care, provision of self-support and community resources $s_{i}$ tracking and coordination of care; ${ }_{i}$ and measurement and improvement of performance. Practices meeting criteria can achieve 1 of 3 levels of recognition. ${ }^{4}$ The NCQA criteria have evolved over time, with initial standards released in 2008 and updated in 2011 and 2014.

Evidence is mixed on how well the PCMH model works, however. In a small randomized controlled trial, the performance of 18 intervention practices, based on the NCQA Physician Practice Connections PCMH model, was compared with that of a control group of 14 practices, measured over a 2-year time period. ${ }^{5}$ Practice performance was evaluated on 11 quality mea- sures based on the Healthcare Effectiveness Data and Information Set, 10 efficiency indicators, and a panel of measures assessing cost of care. Relative to the control group, the intervention group showed modest improvement on a minority of quality and efficiency indicators and reduced emergency department visits, but no cost savings. A larger, 3-year multipayer medical home pilot project that provided financial incentives for achieving NCQA PCMH recognition reported improvement on only 1 of 11 quality measures, and no changes in utilization or costs when measured against those of comparison practices. ${ }^{6}$ And a study that compared costs and utilization among Medicare beneficiaries in practices with and without PCMH recognition found lower total annual Medicare payments, levels of emergency department visits, and acute care hospital payments among PCMH practices, but no differences in hospital admissions or readmissions. ${ }^{7}$ A systematic review by the Agency for Healthcare Research and Quality concluded that the PCMH is a promising, rapidly evolving innovation, but more well-designed studies that evaluate the full PCMH model are needed before drawing firmer conclusions as to the model's effectiveness. ${ }^{8}$

Physicians therefore are faced with the challenge of dealing with multiple change initiatives occurring during a time of considerable uncertainty. To make this dilemma concrete: should physicians spend time and energy focusing on structural measures and process changes such as those embodied in NCQA PCMH recognition? Or should they focus on improving quality and efficiency measures from private payers that could affect their fee schedule, degree of participation in narrow networks, or patient volumes? Or should they focus on making sure they report and improve on measures from Centers for Medicare and Medicaid Services to avoid reductions in fees from the Medicare fee-for-service program?

\section{A CASE STUDY: THE UNITEDHEALTHCARE EXPERIENCE}

\section{UnitedHealthcare Premium Designation}

UnitedHealthcare, a large national health insurer, has been operating a large-scale program to measure and report on physician quality and efficiency performance since 2005. This program, UnitedHealth Premium Designation Program (hereafter, "Premium program"), uses claims and administrative data to assess quality and efficiency performance of physicians across multiple specialties. The program involves nearly 250,000 US physicians, operates in 41 states, and covers 21 medical specialties. The program's approach to measuring quality and efficiency illustrates how many claims-based measurement programs work. 
The Premium program assesses the quality of care physicians provide using more than 300 measures across all specialties (172 for primary care), including those endorsed by the National Quality Forum, NCQA, and others that were developed by medical specialty societies or expert panels and reviewed by committees of practicing physicians. Physicians' performance on quality of care is assessed by identifying specific opportunities to provide evidence-based care, determining whether that care was provided during a given time period ( 1 to 3 years, depending on the measure), aggregating the successes and opportunities attributed to these successes across all eligible rules, and then comparing a physician's success rate with a benchmark.

The Premium program assesses efficiency by measuring case-mix and risk-adjusted costs using a data set that includes fee-for-service claims from the more than 20 million enrollees in UnitedHealthcare's commercial plans each year whose care is not paid via capitation. The data set contains actual costs incurred-that is, allowed costs rather than billed charges-for physician, hospital, pharmacy, and other services. Physicians are compared using the cost of patients (population-based measurement) and episodes of care (episode-based measurement) attributed to them and are measured against benchmarks after adjustment for risk and episode (disease) class, patient severity, physician specialty, geographic area, and patient pharmacy benefit status. More detailed information about the quality measures, rules used to attribute patients and episodes of care to physicians, and other methodologic details is available online. ${ }^{9}$

\section{UnitedHealthcare and PCMH: Longitudinal and Cross-Sectional Experience}

UnitedHealthcare was an early proponent of the PCMH model and developed a number of medical home programs (single-payer and multipayer) across markets in the United States. UnitedHealthcare's experience has been largely favorable in these programs (which have relied on NCQA PCMH certification as a qualification) when measured longitudinally, demonstrating improvement in quality measures for preventive and chronic care, care coordination, access, and patient satisfaction, and in saving approximately $6.2 \%$ of medical costs on average. ${ }^{10}$

We were, however, also interested in looking at PCMH performance on a cross-sectional basis to assess the model outside of a demonstration environment and to see if PCMH-recognized physicians have different levels of quality and efficiency performance as compared with other physicians. By leveraging the national scope of our data, we were able to perform one of the largest descriptive analyses to date of the PCMH model.
Using physician name and National Provider Identifier, we were able to match the Premium program physicians to NCQA PCMH-recognized physicians, resulting in a match of 17,343 unique physicians in primary care (internal medicine, family practice, or pediatrics). We compared this group with 17,323 primary care physicians in the Premium program data set who were not recognized as a PCMH by NCQA.

Looking at quality in this cross-sectional analysis, we found a positive association between achieving Quality in the Premium program and NCQA recognition status, with significantly higher odds of having $\mathrm{PCMH}$ recognition and of passing the Premium program Quality Designation compared with not passing this designation while having PCMH recognition. We also found, however, significantly lower odds that a physician who met botb our Quality and Efficiency criteria would have $\mathrm{PCMH}$ recognition.

As it appeared that $\mathrm{PCMH}$ recognition was positively correlated with better Quality performance, but negatively correlated with combined Quality and Efficiency performance, we further analyzed the association between $\mathrm{PCMH}$ recognition and Efficiency using both $\chi^{2}$ and logistic regression analyses. In these analyses, we found a negative association between meeting the Premium program Efficiency designation criteria and having PCMH recognition, with significantly lower odds that a Premium program Efficiencydesignated physician had $\mathrm{PCMH}$ recognition than a physician not meeting the Efficiency criteria.

\section{Implications for Physicians}

The differences between these longitudinal and crosssectional views of physician performance and their relationship to $\mathrm{PCMH}$ recognition illustrate a number of issues facing physicians today. First, there is unlikely to be a single view of physician performance; rather, there are likely to be multiple views, with different levels of analysis, time frames, and methodologies. These views may or may not point in the same direction and yet may still be "correct."

Second, it is important for physicians to understand and develop the appropriate actions based on the data, and endeavor to avoid both overreaction and underreaction. For example, in our analysis of the association between NCQA PCMH recognition and empirically measured quality and efficiency performance in the Premium program, we found that this recognition was positively associated with achieving Quality benchmarks, but negatively associated with achieving Efficiency benchmarks. Rather than just inferring that the PCMH model is "less efficient" or being overly alarmed that achieving PCMH recognition would negatively affect one's practice, physicians should keep 
several points in mind. It is possible that practices that have achieved NCQA PCMH recognition increase the use of underused services that, although helping them achieve better Quality results, increase comparative episode costs that make them appear to be less efficient. Additionally, it is possible that some PCMH practices are in fact less efficient, perhaps because of practice redesign or other factors that negatively affect workflows, as has been reported with other practice changes such as adoption of EHRs. ${ }^{11,12}$

Third, as the Premium program uses allowed charges, it is possible that groups with higher fee schedules differentially seek or achieve NCQA recognition, with price variation being the major contributor to lower efficiency.

Lastly, it is possible that $\mathrm{PCMH}$ recognition is associated with other factors (such as more comprehensive data capture) that systematically enhance measured quality while reducing comparative episode efficiency. It is also important to keep in mind the emerging results from longitudinal analyses, which tend to consistently show improvement in quality performance, with more mixed results on efficiency.

In addition, any measurement endeavor will have intrinsic limitations. For example, the Premium program is based on data from a commercially insured population and may not be representative of other groups such as those with Medicare or Medicaid, or the uninsured. Also, the quality and efficiency measures in the Premium program do not capture all areas of clinical medicine and were not designed specifically to measure performance of the PCMH model. In particular, measures of expanded access, comprehensiveness of care, and care coordination, all of which are fundamental to high-quality primary care, may not be well captured by currently available quality measures. Finally, episode-based efficiency measurement has limitations, and although the Premium program measures both total population and attributed episode costs, other methods for measuring cost or efficiency could have different patterns of association with $\mathrm{PCMH}$ recognition.

\section{KEY QUESTIONS AND ISSUES FOR PHYSICIANS BEING MEASURED}

Just as in clinical practice, the experienced physician will incorporate multiple perspectives and apply triangulation to develop the best course of action in diagnosis or treatment under conditions of uncertainty, that same approach can be used in understanding and acting on the data from measurement programs.

Key issues physicians should focus on include the following:
- Attribution: Are the patients I'm being measured on "my" patients?

- Scope: Are the scope of services in the measurement program within my influence?

- Comparators: With whom or what am I being compared?

- Reliability/Validity: Does the measurement program include reliable, valid measures?

- Statistical Testing: Does the program include appropriate statistical testing to ensure that measured differences are likely to be real and not random variation?

- Risk adjustment: Does the program adjust appropriately for risk?

- Feedback and Improvement Potential: Does the program do more than measure? Does it offer help to improve? Does it provide specific actionable information for improvement?

\section{CONCLUDING THOUGHTS}

Even as EHRs, clinical registries, and other more fine-grained sources of data evolve and mature, claimsbased measurement is here to stay for the foreseeable future. It is critical that practicing physicians develop a deeper understanding of how they are being measured. Perhaps the most noteworthy first step: be sure to look at your data and the methodology of the measurement program. If there are errors in the measures, reach out to correct them and help improve the measurement program. Then, use the data to work on meaningful improvement.

Those who are doing the measurement, such as payers, need to continue to work to better align measurement approaches to achieve greater consistency, and work to develop common approaches across payers to achieve larger sample sizes. At all levels-the physician, the practice, and the system-major opportunities exist to improve quality and efficiency of care through measurement and improvement.

To read or post commentaries in response to this article, see it online at http://www.annfammed.org/content/13/3/264.

Key words: measurement; patient-centered medical home; quality; efficiency; practice change; health care; primary care; benchmarks; practice-based research

Submitted September 26, 2014; submitted, revised, February 6, 2015; accepted March 2, 2015.

\section{References}

1. Berenson RA, Pronovost PJ, Krumholz HM. Achieving the potential of health care performance measures. Timely Anal Immed Health Pol Iss. 2013;issue 2. http://www.rwjf.org/content/dam/farm/reports/ reports/2013/rwjf406195. Accessed Feb 5, 2015. 
2. Kern LM, Malhotra S, Barrón Y, et al. Accuracy of electronically reported "meaningful use" clinical quality measures: a cross-sectional study. Ann Intern Med. 2013;158(2):77-83.

3. Berenson RA, Rich EC. US approaches to physician payment: the deconstruction of primary care. J Gen Intern Med. 2010;25(6):613-618.

4. National Committee for Quality Assurance. Patient-Centered Medical Home. http://www.ncqa.org/Programs/Recognition/Practices/ PatientCenteredMedicalHomePCMH.aspx. Published 2011. Accessed Feb 6, 2015.

5. Fifield J, Forrest DD, Burleson JA, Martin-Peele M, Gillespie W. Quality and efficiency in small practices transitioning to patient centered medical homes: a randomized trial. J Gen Intern Med. 2013;28(6):778-786.

6. Friedberg MW, Schneider EC, Rosenthal MB, Volpp KG, Werner RM. Association between participation in a multipayer medical home intervention and changes in quality, utilization, and costs of care. JAMA. 2014;311(8):815-825.

7. Van Hasselt M, McCall N, Keyes V, Wensky SG, Smith KW. Total cost of care lower among Medicare fee-for-service beneficiaries receiving care from patient-centered medical homes. Health Serv Res. 2015;50(1):253-272.
8. Peikes D, Zutshi A, Genevro J, Smith K, Parchman M, Meyers D. Early Evidence on the Patient-Centered Medical Home. Final Report. Prepared by Mathematica Policy Research, under Contract Nos. HHSA290200900019l/HHSA29032002T and HHSA290200900019l/ HHSA29032005T. AHRQ Publication No. 12-0020-EF. Rockville, MD: Agency for Healthcare Research and Quality; 2012.

9. UnitedHealth Premium ${ }^{\circledR}$ Physician Designation Program: Detailed Methodology. https://www.unitedhealthcareonline.com/ccmcontent/ ProviderII/UHC/en-US/Assets/ProviderStaticFiles/ProviderStaticFilesPdf/Unitedhealth\%20Premium/Detailed_Methodology_2013-2014. pdf. Published 2014. Accessed Feb 5, 2015.

10. UnitedHealth Center for Health Reform \& Modernization. Advancing Primary Care Delivery. Practical, Proven, and Scalable Approaches. http://www.unitedhealthgroup.com/ /media/UHG/ PDF/2014/UNH-Primary-Care-Report-Advancing-Primary-CareDelivery.ashx. Published Sep 2014. Accessed Feb 5, 2015.

11. Fleming NS, Becker ER, Culler SD, et al. The impact of electronic health records on workflow and financial measures in primary care practices. Health Serv Res. 2014;49(1 Pt 2):405-420.

12. Nguyen L, Bellucci E, Nguyen LT. Electronic health records implementation: an evaluation of information system impact and contingency factors. Int J Med Inform. 2014 Jul 22. [Epub ahead of print].

\section{Get the Annals of Family Medicine by E-mail}

\author{
Make sure you see every new issue \\ while it's fresh; have the table of \\ contents sent to you by e-mail for \\ easy access to articles of interest.
}

Don't miss important research.

Request the e-mail table of contents at

http://www2.highroadsolution.com/

aafp_annals_preference_center/search.aspx

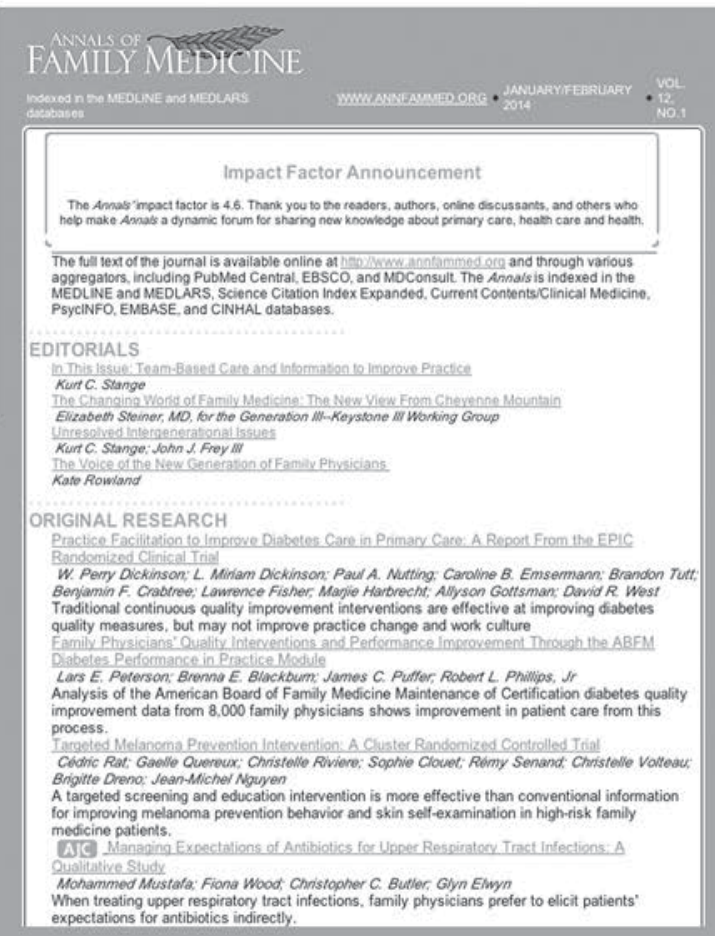

